\title{
Low-temperature phase transitions in some quaternary solid solutions of IV-VI semiconductors
}

\author{
A. I. Lebedev $*$ and I. A. Sluchinskaya \\ Physics Department, Moscow State University, 119991 Moscow, Russia
}

\begin{abstract}
Samples of $\mathrm{PbS}_{x} \mathrm{Se}_{y} \mathrm{Te}_{1-x-y}, \mathrm{~Pb}_{1-x} \mathrm{Sn}_{x} \mathrm{Te}_{1-y} \mathrm{Se}_{y}$, and $\mathrm{Pb}_{1-x} \mathrm{Sn}_{x} \mathrm{Te}_{1-y} \mathrm{~S}_{y}$ quaternary solid solutions were investigated in the $4-200 \mathrm{~K}$ temperature range using electrical and X-ray methods. The regions where low-temperature phase transitions occur were established. It was shown that phase transitions in these solid solutions are associated with off-center S and Sn ions. The dependence of the phase transition temperature on the composition of solid solutions can be qualitatively described taking into account the influence of substitutional disorder on the ordering and tunneling of off-center ions.
\end{abstract}

DOI : 10.1016/0925-8388(94)90713-7 [Journal of Alloys and Compounds 203, 51-54 (1994)]

\section{INTRODUCTION}

IV-VI family narrow-gap semiconductors are widely used for optoelectronic and thermoelectric device fabrication. Although ternary solid solutions are used to tune the spectral characteristics of infrared (IR) lasers and photodetectors, higher performance of these devices can be achieved on heterostructures, which use both ternary and quaternary solid solutions.

The low-temperature ferroelectric phase transitions are interesting features of some IV-VI semiconductors. Such phase transitions were observed in binary GeTe and $\mathrm{SnTe}$ compounds, in $\mathrm{Pb}_{1-x} \mathrm{Sn}_{x} \mathrm{Te}, \mathrm{Sn}_{1-x} \mathrm{Ge}_{x} \mathrm{Te}, \mathrm{Pb}_{1-x} \mathrm{Ge}_{x} \mathrm{Te}$, and $\mathrm{PbTe}_{1-x} \mathrm{~S}_{x}$ ternary and some quaternary solid solutions [1-4]. The changes in the physical properties of these semiconductors induced by phase transition may be interesting for some applications. In particular, the weak temperature dependence of the energy gap near the phase transition temperature $T_{c}$ may be used for the fabrication of continuously tunable lasers [5], and the strong reduction of tunneling currents in $p-n$ junctions near $T_{c}$ may be used to improve the characteristics of photovoltaic detectors [6]. As the temperature of the phase transitions (usually 20-200 K) lies within the working temperatures of IR devices, and there is not much information on low-temperature phase transitions in quaternary solid solutions, it was important to study the low-temperature phase diagrams of quaternary solid solutions in order to find out the regions where the phase transitions can affect the physical properties of solid solutions.

\section{EXPERIMENTAL DETAILS}

Studies of three quaternary solid solutions $\left(\mathrm{Pb}_{1-x} \mathrm{Sn}_{x} \mathrm{Te}_{1-y} \mathrm{Se}_{y}, \quad \mathrm{~Pb}_{1-x} \mathrm{Sn}_{x} \mathrm{Te}_{1-y} \mathrm{~S}_{y}, \quad\right.$ and $\mathrm{PbS}_{x} \mathrm{Se}_{y} \mathrm{Te}_{1-x-y}$ were performed on the samples within the whole region of the stability of the roomtemperature cubic phase. Single crystals as well as

* swan@scon155.phys.msu.ru polycrystalline samples of $n$ - and $p$-type were studied. Single crystals were grown by the sublimation method. Polycrystalline samples were prepared by alloying the appropriate amounts of binary compounds or ternary solid solutions in evacuated silica ampoules with subsequent annealing at $600-710^{\circ} \mathrm{C}$ for $25-220 \mathrm{~h}$. After annealing, the samples were cooled in air or quenched in cold water to prevent the decomposition of the solid solution. In order to obtain samples of $p$ - or $n$-type conductivity, a small amount of excess $\mathrm{Pb}$ or chalcogen was added to the alloys. The homogeneity of the samples was checked by the X-ray method. Most of the investigation was performed on polycrystals in order to know the exact sample composition.

The temperature dependence of resistivity $\rho(T)$ for these crystals was studied in the range 4.2-200 K [7]. Fig. 1] shows typical $\rho(T)$ curves for $\mathrm{PbS}_{x} \mathrm{Se}_{y} \mathrm{Te}_{1-x-y}$ and $\mathrm{Pb}_{1-x} \mathrm{Sn}_{x} \mathrm{Te}_{1-y} \mathrm{Se}_{y}$ samples. The anomalous resistivity peaks associated with the scattering of free carriers by the ferroelectric fluctuations near the phase transition [8] are seen on most of the plots. The $\rho(T)$ curves obtained during heating and cooling (the directions of temperature change are shown by arrows in Fig. 1) differ in a wide temperature range around an anomalous resistivity peak. The difference between the peak positions on heating and cooling was usually $1-3 \mathrm{~K}$, so the phase transition temperature $T_{c}$ was taken as the mean value of the anomalous resistivity peak temperatures. At low temperatures some $\rho(T)$ curves exhibited the enhancement of $\rho$ with decreasing temperature, so-called additional lowtemperature scattering. The causes of the appearance of hysteresis and additional low-temperature scattering accompanying the phase transition in crystals were discussed in detail in our previous papers [4, 9 11].

The phase transitions in $\mathrm{Pb}_{1-x} \mathrm{Sn}_{x} \mathrm{Te}_{y} \mathrm{Se}_{1-y}$ and $\mathrm{Pb}_{1-x} \mathrm{Sn}_{x} \mathrm{Te}_{1-y} \mathrm{~S}_{y}$ crystals were studied by a lowtemperature X-ray technique, which revealed the rhombohedral distortion of the structure below $T_{c}$.

Figures 24 show the dependence of $T_{c}$ on the composition of three quaternary systems $(\mathrm{Pb}-\mathrm{S}-\mathrm{Se}-\mathrm{Te}, \mathrm{Pb}-$ $\mathrm{Sn}-\mathrm{Te}-\mathrm{Se}$ and $\mathrm{Pb}-\mathrm{Sn}-\mathrm{Te}-\mathrm{S})$. The points in these figures correspond to compositions of samples. The solid curves are isotherms drawn after computer approximation of the 

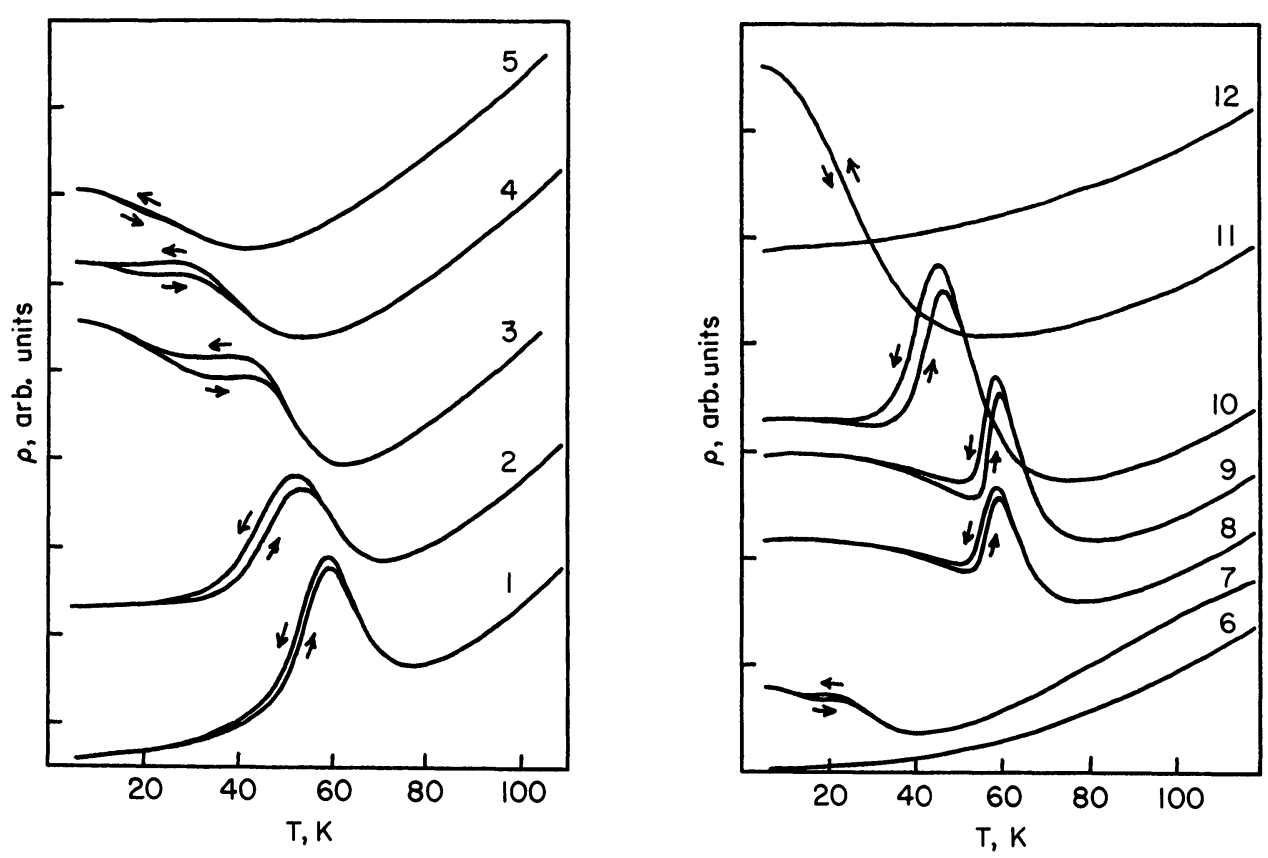

FIG. 1. Typical $\rho(T)$ plots for $\mathrm{PbS}_{x} \mathrm{Se}_{y} \mathrm{Te}_{1-x-y}$ and $\mathrm{Pb}_{1-x} \mathrm{Sn}_{x} \mathrm{Te}_{1-y} \mathrm{Se}_{y}$ solid solutions. Curves 1-5 correspond to the samples of $\mathrm{PbS}_{x} \mathrm{Se}_{y} \mathrm{Te}_{1-x-y}$ with constant $x=0.08$ and $y=0.21,0.27,0.34,0.40$, and 0.46 , respectively. Curves $6-12$ correspond to the samples of $\mathrm{Pb}_{1-x} \mathrm{Sn}_{x} \mathrm{Te}_{1-y} \mathrm{Se}_{y}$ with constant $x=0.2$ and $y=0,0.05,0.25,0.33,0.5,0.67$, and 0.83 , respectively. The curves are shifted arbitrarily along the vertical axis. The arrows show the direction of the temperature change during recording the curves.

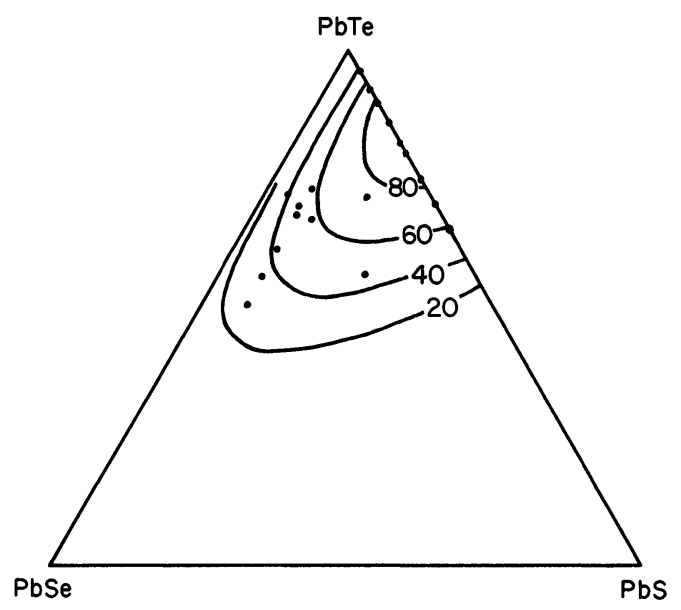

FIG. 2. The dependence of $T_{c}$ (in K) upon composition of the $\mathrm{PbS}_{x} \mathrm{Se}_{y} \mathrm{Te}_{1-x-y}$ solid solution.

experimental data.

\section{DISCUSSION}

Our results show that in all three systems there are regions where the low-temperature phase transitions occur.
The manifestations of the phase transition in the electrical properties of these solid solutions were similar to that in ternary $\mathrm{Pb}_{1-x} \mathrm{Ge}_{x} \mathrm{Te}$ and $\mathrm{PbTe}_{1-x} \mathrm{~S}_{x}$ solid solutions, where the phase transitions are unambiguously associated with the presence of off-center ions [12, 13]. This enabled us to suppose that phase transitions in investigated quaternary solid solutions have the same origin and are induced by off-center ions. Following the results of Ref. [3, one could suppose that the $\mathrm{S}$ atom is off-center in $\mathrm{PbS}_{x} \mathrm{Se}_{y} \mathrm{Te}_{1-x-y}$, while according to Refs. 10 and 11, $\mathrm{Sn}$ is off-center in $\mathrm{Pb}_{1-x} \mathrm{Sn}_{x} \mathrm{Te}_{1-y} \mathrm{Se}_{y}$, and $\mathrm{Sn}$ and $\mathrm{S}$ are both off-center in $\mathrm{Pb}_{1-x} \mathrm{Sn}_{x} \mathrm{Te}_{1-y} \mathrm{~S}_{y}$.

The individual features of off-center ions and host crystals, into which off-center ions are incorporated, result in a different dependency of $T_{c}$ on composition. Fig. 2 shows that maximum values of $T_{c}$ on the $\mathrm{PbS}_{x} \mathrm{Se}_{y} \mathrm{Te}_{1-x-y}$ phase diagram are achieved in ternary $\mathrm{PbTe}_{1-x} \mathrm{~S}_{x}$ solid solutions; in other ternary solid solutions $\left(\mathrm{PbS}_{1-x} \mathrm{Se}_{x}\right.$ and $\left.\mathrm{PbTe}_{1-x} \mathrm{Se}_{x}\right)$ there are no phase transitions. This emphasizes the key role of substitution of Te by $\mathrm{S}$ in the appearance of phase transitions in $\mathrm{PbS}_{x} \mathrm{Se}_{y} \mathrm{Te}_{1-x-y}$. The region where phase transitions take place is located near $\mathrm{PbTe}$ whose lattice is known to be softest among the different lead chalcogenides. Adding Se to $\mathrm{PbTe}_{1-x} \mathrm{~S}_{x}$ lowers $T_{c}$; one of the causes of this lowering may be the influence of random fields (resulting from the substitutional disorder in the quaternary solid solution) on the ordering of off-center ions [4]. 


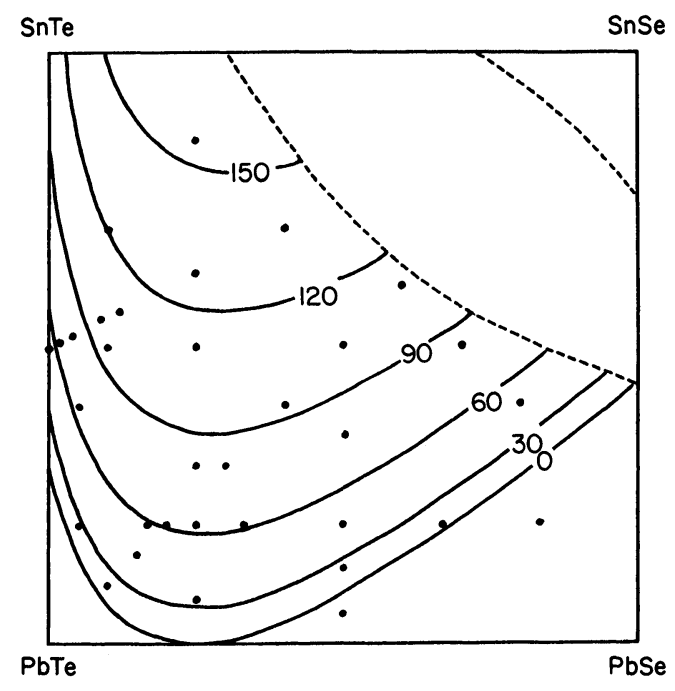

FIG. 3. The dependence of $T_{c}$ (in K) upon composition of the $\mathrm{Pb}_{1-x} \mathrm{Sn}_{x} \mathrm{Te}_{1-y} \mathrm{Se}_{y}$ solid solution. The dotted lines show the stability limits for the solid solution.

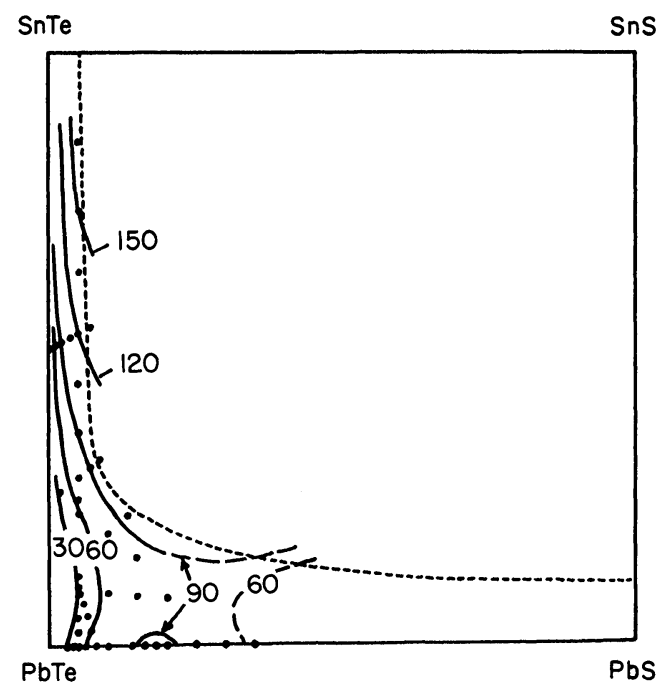

FIG. 4. The dependence of $T_{c}$ (in K) upon composition of the $\mathrm{Pb}_{1-x} \mathrm{Sn}_{x} \mathrm{Te}_{1-y} \mathrm{~S}_{y}$ solid solution. The dotted line shows the stability limits for the solid solution according our data.

A qualitatively different behavior was observed in $\mathrm{Pb}_{1-x} \mathrm{Sn}_{x} \mathrm{Te}_{1-y} \mathrm{Se}_{y}$ solid solution. As follows from the phase diagram shown in Fig. 3, the substitution of Te by $\mathrm{Se}$ in $\mathrm{Pb}_{1-x} \mathrm{Sn}_{x}$ Te crystals with low tin concentration $(x<0.35)$ results in the onset of the phase transition. When increasing Se concentration in crystals with constant $x$, the phase transition temperature first increased quickly, then reached a maximum (at about $y=0.25$ ), and decreased again down to zero when approaching $\mathrm{Pb}_{1-x} \mathrm{Sn}_{x}$ Se. At constant $y=0.25$, the phase transition could be observed starting from Sn concentrations as low as $x=0.08$. According to our computer approximation of the experimental data, the maximum value of $T_{c}$ should be achieved near $x=1$ and $y=0.25$, but another phase transition took place in $\mathrm{SnTe}_{0.75} \mathrm{Se}_{0.25}$ at a higher temperature [10]. The maximum value of $T_{c}$ in this quaternary solid solution was 1.5 times higher than in the best single crystals of SnTe [14]. The unusual rise of $T_{c}$ with increasing the substitutional disorder in $\mathrm{Pb}_{1-x} \mathrm{Sn}_{x} \mathrm{Te}_{1-y} \mathrm{Se}_{y}$ was explained in Ref. 10: the substitutonal disorder does not only produce random fields, but it can depress strongly the tunneling motion of off-center ions and so may result in an increase of $T_{c}$. We suppose that the latter effect is predominant in $\mathrm{Pb}_{1-x} \mathrm{Sn}_{x} \mathrm{Te}_{1-y} \mathrm{Se}_{y}$.

$\mathrm{Pb}_{1-x} \mathrm{Sn}_{x} \mathrm{Te}_{1-y} \mathrm{~S}_{y}$ is the most interesting system studied in this work. There are two different off-center ions in this system, S and Sn. As follows from Fig. 4 their simultaneous effect on $T_{c}$ is more complicated than in $\mathrm{Pb}_{1-x} \mathrm{Sn}_{x} \mathrm{Te}_{1-y} \mathrm{Se}_{y}$. The substitution of small amounts of $\mathrm{Pb}$ by $\mathrm{Sn}$ in crystals with fixed $\mathrm{S}$ concentration first strongly decreased $T_{c}$ so that the phase transition disappeared at about $x \approx 0.1$. With further increasing Sn concentration, the phase transition appeared again. Thus, the low-temperature phase diagram consists of two regions; in one of these (at low Sn concentration) the ferroelectric phase is induced by $\mathrm{S}$ off-center ions, and in the other it is associated with Sn off-center ions. The phase diagram is so complicated (it even has a saddle point) because the substitutional disorder depresses the ordering of S off-center ions (by random fields) and at the same time enhances the cooperative motion of Sn off-center ions due to decreasing their tunneling.

The comparison of phase diagrams in Figs. 3 and 4 shows that they are similar in the region of high $\mathrm{Sn}$ concentration. The substitution of Te by $\mathrm{S}$ results in a stronger increase of $T_{c}$ than in the case of substituting Te by Se; we suppose that it is due to a stronger perturbation produced in the former case. The similarity between these phase diagrams confirms that the origin of the rise of $T_{c}$ in both cases is the same - the depression of the tunneling motion of Sn off-center ions by the substitutional disorder [11]. Unfortunately, the composition stability limits for $\mathrm{Pb}_{1-x} \mathrm{Sn}_{x} \mathrm{Te}_{1-y} \mathrm{~S}_{y}$ are not very large; the boundaries of the single phase region (according to our data) are indicated by the dotted line in Fig. 4.

\section{CONCLUSIONS}

1. The samples of $\mathrm{PbS}_{x} \mathrm{Se}_{y} \mathrm{Te}_{1-x-y}$, $\mathrm{Pb}_{1-x} \mathrm{Sn}_{x} \mathrm{Te}_{1-y} \mathrm{Se}_{y}$, and $\mathrm{Pb}_{1-x} \mathrm{Sn}_{x} \mathrm{Te}_{1-y} \mathrm{~S}_{y}$ quaternary solid solutions have been studied at low temperatures using electrical and X-ray methods. The regions where the low-temperature phase transitions take place were established.

2. It was shown that the low-temperature phase transitions in all three systems are associated with off-center $\mathrm{S}$ and $\mathrm{Sn}$ ions. 
3. The dependence of the phase transition temperature on the composition of solid solutions may be quali- tatively described by taking into account the influence of substitutional disorder on the ordering and tunneling of off-center ions.

[1] W. Jantsch, Dielectric properties and soft and impurities on the carrier scattering near the phase modes in semiconducting $(\mathrm{Pb}, \mathrm{Sn}, \mathrm{Ge}) \mathrm{Te}$, in transition in ferroelectric semiconductor $\mathrm{Pb}_{1-x} \mathrm{Ge}_{x} \mathrm{Te}_{\mathrm{e}}$ Dynamical Properties of IV-VI Compounds. Springer Tracts in ModerRhylsysolid State 25, 2055 (1983). Vol. 99 (Springer-Verlag Berlin Heidelberg, 1983) pp. $1-50$.

[2] K. Murase, The structural phase transition in IV-VI semiconductors, J. Phys. Soc. Jpn. 49 Suppl. A, 725 (1980).

[3] K. A. Abdullin, A. I. Lebedev, A. M. Gas'kov, V. N. Demin, and V. P. Zlomanov, Structural phase transition in the $\mathrm{PbTe}_{1-x} \mathrm{~S}_{x}$ solid solution, JETP Lett. 40, 998 (1984).

[4] A. I. Lebedev and I. A. Sluchinskaya, Phase transitions in $\mathrm{Pb}_{1-x} \mathrm{Ge}_{x} \mathrm{Te}_{1-y} \mathrm{~S}_{y}$ solid solution induced by ordering of (Ge, S) off-center impurities, Izv. Akad. Nauk USSR, Ser. Fiz. 51, 1683 (1987).

[5] G. A. Antcliffe, S. G. Parker, and R. T. Bate, cw operation and nitric oxide spectroscopy using diode lasers of $\mathrm{Pb}_{1-x} \mathrm{Ge}_{x} \mathrm{Te}, \mathrm{Appl}$. Phys. Lett. 21, 505 (1972)

[6] S. P. Grishechkina, S. V. Zhokhovets, B. D. Kopylovskii, and A. P. Shotov, Influence of a ferroelectric phase transition on electrical characteristics of $p$ - $n$-junctions in $\mathrm{Pb}_{1-x} \mathrm{Ge}_{x} \mathrm{Te}$, Sov. Phys. Semicond. 12, 671 (1978).

[7] K. A. Abdullin and A. I. Lebedev, Influence of defects
[8] K. L. I. Kobayashi, Y. Kato, Y. Katayama, and K. F. Komatsubara, Resistance anomaly due to displacive phase transiton in SnTe, Solid State Commun. 17, 875 (1975).

[9] A. I. Lebedev and I. A. Sluchinskaya, Possible appearance of a dipole-glass phase in solid solutions of IV-VI group semiconductors, JETP Lett. 46, 536 (1987).

[10] A. I. Lebedev and I. A. Sluchinskaya, Structural disorder and phase transition in $\mathrm{Pb}_{1-x} \mathrm{Sn}_{x} \mathrm{Te}_{1-y} \mathrm{Se}_{y}$, Sov. Phys. Solid State 32, 1036 (1990).

[11] A. I. Lebedev and I. A. Sluchinskaya, New arguments for off-centering of $\mathrm{Sn}$ atoms in $\mathrm{Pb}_{1-x} \mathrm{Sn}_{x} \mathrm{Te}_{1-y} \mathrm{Se}_{y}$ and $\mathrm{Pb}_{1-x} \mathrm{Sn}_{x} \mathrm{Te}_{1-y} \mathrm{~S}_{y}$, Sov. Phys. Solid State 34, 793 (1992).

[12] Q. T. Islam and B. A. Bunker, Ferroelectric transition in $\mathrm{Pb}_{1-x} \mathrm{Ge}_{x} \mathrm{Te}$ : Extended x-ray-absorption fine-structure investigation of the $\mathrm{Ge}$ and $\mathrm{Pb}$ sites, Phys. Rev. Lett. 59, 2701 (1987).

[13] B. A. Bunker, Z. Wang, and Q. Islam, XAFS investigations of ferroelectric IV-VI semiconductors, Ferroelectrics 120, 23 (1991).

[14] K. L. I. Kobayashi, Y. Kato, Y. Katayama, and K. F. Komatsubara, Carrier-concentration-dependent phase transition in SnTe, Phys. Rev. Lett. 37, 772 (1976) 